\title{
A Review of Class I and Class II Pet Food Recalls Involving Chemical Contaminants from 1996 to 2008
}

\author{
Wilson Rumbeiha • Jamie Morrison
}

Published online: 2 December 2010

(C) American College of Medical Toxicology 2010

\begin{abstract}
Commercial pet food in USA is generally safe, but adulteration does occur. Adulterated food has to be recalled to protect pets and public health. All stakeholders, including food firms, distributors, and government agencies such as the Food and Drug Administration (FDA) participate in food recall. The objective of this review is to describe the pet food recall procedure from start to finish, and to review class I and II pet food recalls from 1996 to 2008 , with a specific focus on those due to chemical contaminants/adulterants. Information was requested from the FDA by Freedom of Information Act. Only those recalls backed by the FDA scientific review were considered. The legal framework for food recalls in the Code of Federal Regulations, Title 21, Chapter 1, Part 7 and in the Food and Drug Administration Amendments Act of 2007, Title X was reviewed. From 1996 to 2008, there were a total of 22 class I and II pet food recalls. Of these, only six (27\%) were due to chemical adulterants. The adulterants were aflatoxins, cholecalciferol, methionine, and melamine, and cyanuric acid. The causes of adulteration included inadequate testing of raw materials for toxins, use of wrong or faulty mixing equipment, and misformulation of raw materials.
\end{abstract}

J. Morrison

College of Veterinary Medicine, Michigan State University,

East Lansing, MI, USA

\section{W. Rumbeiha}

Department of Pathobiology and Diagnostic Investigation, College of Veterinary Medicine, Michigan State University, East Lansing, MI, USA

W. Rumbeiha $(\square)$

DCPAH, CVM, Michigan State University,

4125 Beaumont Road,

East Lansing, MI 48910-8104, USA

e-mail: Rumbeiha@dcpah.msu.edu
Overall, pet food manufactured in the USA is safe. Even with shortcomings in the recall process, the incidence of illness associated with pet food adulteration is low. Added changes can only make the system better in the future to safeguard pet and public safety.

Keywords Recall process $\cdot$ Pet food recall $\cdot$ Class I and II recall $\cdot$ Food adulteration

\section{Introduction}

With approximately 77.5 million pet dogs and 93.6 million pet cats in the USA [1], over 8,011,774.9 $\mathrm{t}$ of dog and cat food is produced [2]. Commercial pet food in USA is generally safe. Less than $1.7 \%$ of reported food poisonings in pets are related to pet food [3]. Unfortunately, pet food adulteration does occur from time to time. The actual number of pets affected by adulterated pet food is not known because hitherto there was no adverse reporting system for pet foods. However, estimated numbers are in the hundreds [4]. The objectives of this review are: (1) to describe the pet food recall process from start to finish, and (2) to review pet food recalls from 1996 to 2008 in the USA that were triggered by presence of chemical adulterants. Food recall occurs both for human and pet foods. Although pet food is made for pets, it has been documented that people with limited resources eat pet food [5]. Therefore when pet food is adulterated it has to be recalled to protect pet and public health. The procedure of pet food recall is contained in the Code of Federal Regulations Title 21 (CFR 21) Chapter 1, Part 7 [6]. Because of the shortcomings exposed by the melamine pet food recall of 2007, a new law called The Food and Drug Administration, Title X of 2007 was passed in order to strengthen the food recall process [7]. 


\section{The Pet Food Recall Process}

The process of pet food recall is complex. Currently the laws governing the food recall process are spelled out in CFR 21 Chapter 1, Part 7. Section 7.40 spells out the recall procedure in detail [6]. According to this section, a recall is a method of removing or correcting consumer products that are in violation of laws administered by the Food and Drug Administration (FDA). A recall is an alternative to the FDA-initiated court action to remove or correct consumer products that are in violation of the FDA laws. A recall may be initiated in one of two ways. First, a recall can be voluntarily initiated by the manufacturer (firm) and distributors of the consumer products which are in violation of the FDA laws. Alternatively, a voluntary recall can be undertaken by the firm at the request of the FDA. The FDA conducts analytical surveys on food and when adulteration is confirmed the agency sends a notice to the firm asking them to conduct a recall. In case the firm refuses to undertake a recall, or if the recall was not effective, the FDA can issue a court order to the firm to recall the food. This would be called an involuntary food recall. In practice, there is little incentive for firms to refuse an FDA request for a recall. This is primarily because of the high costs associated with litigation and the bad publicity that would befall a company that refuses to undertake a voluntary recall of a product determined to be in violation of FDA regulation [3].

The recall process starts with gathering information about the product in order to evaluate the problem. This includes evaluation of consumer complaints called in to the consumer complaint department of the firm and/or the FDA. Once complaints are lodged with the firm, the latter conducts its investigations and may issue a voluntary food recall. According to Section 7.46, a firm initiates a recall if it believes the product to be in violation of the federal laws and will immediately notify the FDA. Such removal or correction is considered a recall only if the FDA determines the product involving a violation is subject to legal action [5]. The new Food and Drug Administration Amendment (FDAA) Act of 2007 now requires that firms submit a report to the FDA no later than $24 \mathrm{~h}$ after determining that there is a reasonable probability that the use or exposure to the food will cause serious adverse health consequences or death to humans or animals [6]. Following consumer complaints to the FDA or notification by the firm, the agency collects more data through interview of the complainant(s), collects and analyses pet food samples at approved FDA laboratories. This data is reviewed by an ad hoc scientific review committee of the FDA. This process is called a Health Hazard Evaluation (HHE). A comprehensive assessment is done including whether any disease or injuries have occurred from use of the product. The panel also assesses the seriousness of the health hazard, the likelihood of occurrence and of the consequences of the health hazard. This information is used to classify the recall to indicate the seriousness of the health hazard and also to formulate the recall strategy with respect to the depth of the recall according to CFR 21 Chapter 1 Section 7.41 [6]. A class I recall is the most severe and involves food in which there is reasonable probability that it will cause serious adverse health consequences or death. A class II recall involves a food in which temporary or medically reversible adverse health consequences may result but the probability of serious adverse health consequences are remote. A class III recall involves a food which is not likely to cause adverse health consequences [6]. As for the recall strategy, the depth of the recall can be set at one of three levels, i.e., at the consumer or user level, at the retail level, or at wholesale level [6].

The bulk of the recall work is shouldered by the firm, with the FDA overseeing each and every step of the process as executed by the firm. In addition the FDA, in consultation with the firm, will publicize the recall via general public warnings or warnings through specific segments of the population such as veterinarians. The recalling firm is also responsible for conducting effectiveness checks to ascertain that all parties to the recall depth have received notification about the recall. The FDA has written guidelines for effectiveness checks and these are available to recalling firms upon request from the FDA.

Once a recall is completed it has to be terminated. Steps involved in termination of a recall are given in CFR21 Chapter 1 Section 7.55 [6]. The FDA will terminate the recall when it is satisfied that all reasonable efforts have been made to remove the product in accordance with the strategy, and that the product has been removed and properly disposed of. Termination is by way of written communication from the FDA to the firm. Alternatively, the recalling firm may take the initiative to request the FDA to terminate the recall. In this case, the written request to the FDA is accompanied by the most current recall status report and a description of the disposition of the product.

Because the melamine pet food recall of 2007 exposed many deficiencies in the recall process, congress passed the Food and Drug Administration Amendment Act (FDAAA) of 2007 [7]. This law puts the burden on the FDA to improve the food recall process. The law required that within 1 year of enactment, the FDA shall establish an early warning and surveillance system to identify adulteration of pet-food supply and outbreaks of illness associated with pet food. With this system in place, it will likely be easier to track pet cases and to determine the burden on pet health arising from consuming adulterated pet food. The law also required the establishment of a single searchable website of recalled pet food, improvement in coordination and 
collaboration with states, and the creation of a reportable food registry where adverse pet food events can be reported. The law also put new demands on firms. It puts the burden on the firm to report to the FDA and to investigate the cause of adulteration of the pet food within $24 \mathrm{~h}$ of learning about it. This new requirement will cut "dead time" from when the firms first know about the problem with pet food to the time they report it to the FDA. This will enhance communication and likely minimize the impact on pet health. Presently, the FDA has an active Reportable Food Registry which is accessible at http:// www.safetyreporting.hhs.gov. They also have a website educating consumers on how to report a pet food complaint which is accessible at http://www.fda.gov/animalveterinary/ safetyhealth/reportaproblem/ucm182403.htm. The FDA consumer complaint reporting system is currently the agency's surveillance tool for pet-food early warning system. Consumers can report pet food complaints at the FDA's 24-h hot line at 301-443-1240. Alternatively, consumers may report complaints to geographic region contacts. The contact information for these contacts is available online at http://www.fda.gov/Safety/ReportaProblem/ ConsumerComplaintCoordinators/default.htm. At present, a single FDA searchable website of recalled pet food is not yet in place. Nonetheless, when it is in place it will significantly improve communication about recalled foods. Ultimately, when all these new changes are fully implemented, the recall process will be tremendously improved.

\section{A Review of Class I and II Pet Food Recalls from 1996 to 2008, with a Specific a Focus on Those Due to Chemical Contaminants/Adulterants}

\section{Methods}

In order to work with credible data, a formal written request was sent to the FDA through the Freedom of Information Act to obtain HHE for class I and II pet food recalls during the period of 1996-2008. Only those HHE related to recalls involving adulteration were requested. Once received, the FDA data critically reviewed to determine the chemical adulterants and the causes of the adulteration. Then a review of pet cases affected by the adulterants in the literature was conducted to determine whether the clinical signs of affected pets were in concordance with those reported in the FDA health hazard evaluations.

\section{Results}

Between 1996 and 2008 there was a total of 22 class I and II pet food recalls documented by the FDA involving adulteration. Of these, only six (27\%) were initiated due to chemical contamination/adulteration (Table 1). Two of the six recalls were due to aflatoxin contamination which occurred in 1998 and 2005-2006, respectively [8]. Two recalls were due to excess vitamin $\mathrm{D}_{3}$ (cholecalciferol) which occurred in 1999 and 2006, respectively [9, 10]. One recall was triggered by confirmed presence excess methionine in dog food [11]. The latest and the largest pet food recall in US history occurred in 2007 and was due to melamine and cyanuric acid contamination [12]. All recalls reported here were firm initiated and voluntary. The recall classification for each recall is shown in Table 1. With regard to strategy, the depth of recalls was level 1 (consumer or use level) for all recalls reported here.

\section{Aflatoxin-Associated Recalls}

Aflatoxins are natural mycotoxins produced both in the field and in storage by many species of fungi. Aflatoxins include $B_{1}, B_{2}, G_{1}$, and $G_{2}$ with $B_{1}$ being the most toxic. In October and November of 1998, a recall was initiated for $1,362,516$ bags of 54 pet food brands and treats made by Doane Products Co., Inc. due to aflatoxin contamination [8]. The aflatoxin contamination of the pet food was attributed to a contaminated batch of corn which was not

Table 1 A chronological summary of class I and II pet food recalls involving chemical adulteration in the USA between 1996 and 2008.

\begin{tabular}{|c|c|c|c|c|}
\hline Year of recall & Product recalled & Reason for recall & $\begin{array}{l}\text { Adverse health effects } \\
\text { reported }\end{array}$ & $\begin{array}{l}\text { Recall } \\
\text { classification }\end{array}$ \\
\hline 1998 & Doane Products Co., Inc. Dry dog food & Aflatoxin contaminated corn & Death of at least 25 dogs & I \\
\hline 1999-2000 & Golden Sun Feeds, Inc. Dry dog food & Excess vitamin $\mathrm{D}_{3}$ & $\begin{array}{l}\text { At least } 7 \text { dead and } 3 \text { illnesses } \\
\text { in dogs }\end{array}$ & I \\
\hline 2001 & The IAMS Company Dry dog food & Excess methionine & Illness in 21 dogs & $\mathrm{n} / \mathrm{a}$ \\
\hline $2005-2006$ & Diamond dog and cat foods & Aflatoxin contamination & At least 100 pets affected & $\mathrm{n} / \mathrm{a}$ \\
\hline 2006 & Royal Canin Veterinary Diet & Excess vitamin $\mathrm{D}_{3}$ & Illness in 6 dogs and 5 cats & II \\
\hline 2007 & $\begin{array}{l}>100 \text { brand names of dog and cat food } \\
\text { manufactured by Menu Foods }\end{array}$ & $\begin{array}{l}\text { Contaminated with the industrial } \\
\text { chemicals melamine and cyanuric acid }\end{array}$ & $\begin{array}{l}\text { At least } 424 \text { dogs and cats } \\
\text { affected [4] }\end{array}$ & I \\
\hline
\end{tabular}

$n / a$, not available 
appropriately tested. The corn had been examined with a black light prior to processing. Black light only detects kojic acid, a common metabolite of toxic fungi, and does not detect aflatoxins in food. Retrospective testing with aflatoxin-specific procedures revealed that four loads of corn used in the manufacture of the recalled food contained aflatoxin in concentrations ranging from 66.4 to $495.3 \mathrm{ppb}$. Dog food consumed by ill or deceased dogs contained aflatoxin concentrations in the range of 35$191 \mathrm{ppb}$. According to the FDA HHE report, at least 25 dog deaths resulted from consumption of the contaminated product [8].

Illness and death in over 100 dogs due to an aflatoxin contamination event involving 19 products of the Diamond brand of dog food occurred in late 2005-early 2006 [13, 14]. Clinical signs in affected dogs included anorexia, lethargy, vomiting, jaundice, and diarrhea [15]. Necropsy revealed enlarged, pale yellow livers, cirrhosis, chronic hepatopathy, hepatic lipidosis, portal fibroplasias, and biliary hyperplasia. Analysis of the feed yielded aflatoxin $\mathrm{B}_{1}$ in range of 223-579 $\mathrm{ppb}$. In livers of affected dogs, the concentration of aflatoxin $\mathrm{M}_{1}$ metabolite of aflatoxin $\mathrm{B}_{1}$ was determined to be in the range of $0.6-4.4 \mathrm{ppb}[13,14]$. The two pet food aflatoxin adulteration events happened because there was inadequate testing of raw materials, particularly corn, which went into manufacturing of pet food. It is therefore vital that firms put in place procedures to adequately test raw materials for presence of aflatoxins to prevent such adulterations from occurring.

As reviewed by Boermans and Leung [16], aflatoxins are the most common mycotoxins to cause acute illness linked to dog food in the world. Past reports of dog aflatoxin toxicosis in the USA include contaminated dog food due to a cornmeal ingredient containing $511 \mathrm{ppb}$ aflatoxin [17]. The FDA has an action level of $20 \mathrm{ppb}$ aflatoxin in dog and cat feed as well as many human food products [18]. Aflatoxins are hepatotoxic, immunosuppressive, nephrotoxic, and carcinogenic $[19,20]$. The acute $\mathrm{LD}_{50}$ of aflatoxin $\mathrm{B}_{1}$ in dogs is $0.5-1.0 \mathrm{mg} / \mathrm{kg}$ and in cats is $0.3-0.6 \mathrm{mg} / \mathrm{kg}$. Acute aflatoxicosis occurs at aflatoxin $B_{1}$ concentrations $\geq 1 \mathrm{ppm}$ in the diet. Chronic aflatoxicosis can result when dogs eat food containing 50-300 ppb over a period of 6-8 weeks [19].

Aflatoxins also cause illness in humans. Children are particularly susceptible. Outbreaks of human acute toxicosis have been reported in India and Kenya with signs of jaundice, ascites, portal hypertension, and high mortality rate [21-23]. Individual accounts of human aflatoxin toxicity include a suicide attempt with ingestion of aflatoxin that resulted in a transient rash, nausea, and headache [24]. Human chronic exposure to aflatoxins has been associated with an increased risk for liver and lung cancer, immunosuppression, and decreased growth rate [25].
Vitamin $\mathrm{D}_{3}$-Associated Recalls

Vitamins are essential nutrients and are added to pet food to meet nutritional needs of pets. There were two pet food recalls resulting from presence of excessive amounts of vitamin $\mathrm{D}_{3}$. In December of 1999, DVM Nutri-Balance High Protein Dog Food and Golden Sun Feeds Hi-Pro Hunter Dog Food were recalled due to presence of excessive amounts of vitamin $\mathrm{D}_{3}$. According to the FDA HHE report, this problem was caused by a feed-mixing error [9]. An inappropriate feed mixer was used which failed to dispense the vitamin uniformly though out the food batch. This error was the cause of illness and deaths in at least 25 dogs. Although the offending food was not available for testing, clinical signs of ill and dead dogs were consistent with vitamin $\mathrm{D}_{3}$ toxicosis. Two animals demonstrated elevated serum calcium. It is estimated that the feedmixing error resulted in an exposure of $14.65 \mathrm{mg} / \mathrm{kg} \mathrm{BW}$ vitamin $\mathrm{D}_{3}$ in the dogs [9].

Subsequent to the 1999 recall, excess vitamin $\mathrm{D}_{3}$ in pet food led to the recall of four products of ROYAL CANIN Veterinary Diet ${ }^{\mathrm{TM}}$ in February of 2006 [10]. According to the FDA HHE report, the excess nutrient was caused by a misformulated vitamin premix containing between 12,230 and $284,700 \mathrm{IU}$ vitamin $\mathrm{D}_{3} / \mathrm{kg}$ instead of the desired $11,820 \mathrm{IU} / \mathrm{kg}$ premix [10]. Concentrations of vitamin $\mathrm{D}_{3}$ in the finished pet food measured between 1,509 and 2,664 IU/1,000 kcal metabolizable energy (ME). The maximum amount of vitamin $\mathrm{D}_{3}$ allowed by AAFCO guidelines in dog and cat food respectively are 1,429 IU/ $1000 \mathrm{kcal} \mathrm{ME}$ and 2,500 IU/1,000 kcal/ME, respectively. Analytical results from the Royal Canin confirmed the presence of excessive amount of the vitamin according to the FDA HHE report. Six dogs and five cats were reported have shown clinical signs consistent with vitamin $D_{3}$ toxicosis including anorexia, lethargy, polyuria/polydipsia, and elevated serum calcium [10]. There was also elevated serum 25-hydroxy cholecalciferol, a definitive marker of vitamin $\mathrm{D}_{3}$ toxicosis [26]. This data shows that the use of inappropriate feed mixing equipment and misformulation can be a source of adulteration in pet food. Pet food firms should put in place safeguards to prevent these kinds of errors.

According to the National Research Council, clinical signs in dogs intoxicated with Vitamin $\mathrm{D}_{3}$ include vomiting, diarrhea, anorexia, depression, weakness, reduced growth rate, loss of condition, hypercalcemia, calcification of soft tissue, and excessive mineralization of long bones, dry and brittle hair, muscular atrophy, coma, puppies stop nursing and become lethargic then die, decreases renal function, and parathyroid cell atrophy [27]. Similar clinical signs were also observed by Rumbeiha et al. in dogs with experimentally induced cholecalciferol poisoning [26]. 
Humans are also susceptible to hypervitaminosis D. The tolerable upper intake levels for vitamin $\mathrm{D}$ in children ages $0-12$ months is $1,000 \mathrm{IU}$ or $25 \mu \mathrm{g} /$ day and for ages 1 year and up is $2,000 \mathrm{IU}$ or $50 \mu \mathrm{g} /$ day. Signs of toxicosis in humans include weakness, headache, anorexia, vomiting, diarrhea, nephrocalcinosis, impaired renal function leading to polyuria, polydipsia, decreased growth in children, weight loss, metastatic calcification, and seizures [28].

\section{Methionine-Associated Recall}

One of the pet food recalls involved adulteration with an amino acid, methionine. In 2000, IAMS Large Breed Formula premium food for adult dogs was recalled due to presence of excessive amount of this amino acid. According to the FDA HHE report [11], following consumer complaints the firm analyzed batches of feed and the concentration of methionine in the dog food was found to be in the range of $1.60-2.75 \%$. This is six times higher than the AAFCO recommended maximum of $0.43 \%$ methionine plus cystine for adult dogs. Anorexia and vomiting were reported in 21 dogs which ate this adulterated food [11].

Methionine is one of the most-limiting amino acids in feeds and is also considered one of the most toxic of the essential amino acids [29]. AAFCO recommends 0.53\% methionine-cystine for growth and reproduction and $0.43 \%$ for adult maintenance based on dry matter in a 3,500 kcal $\mathrm{ME} / \mathrm{kg}$ food for the dog and $1.10 \%$ methionine-cystine for all life stages based on dry matter in a 4,000 kcal ME/kg food for the cat [30]. Methionine and cystine are often considered together as methionine is a precursor to cystine. Both are sulfur containing amino acids. In laboratory experiments, feeding methionine at levels three times higher than recommended levels to young rats caused growth retardation, and injury of the pancreas, liver, and kidney [31]. In a previously published case report, six hunting dogs poisoned by methionine exhibited ataxia, disorientation, hyperactivity, lethargy, tremors, vomiting, and ptyalism [32]. Kittens with an exposure of 6 weeks to $2 \%$ dietary methionine or $0.6 \mathrm{~g}$ methionine $/ \mathrm{kg} \mathrm{BW}$ had a negative effect on the body weight gain and growth rate [33].

In humans, cardiovascular diseases, including arteriosclerosis and thromboembolisms have been reported following excessive methionine intake leading to elevated homocystine, a product of methionine metabolism [34, 35]. A more extensive review of methionine toxicosis in humans was done by Garlick [36].

\section{Melamine and Cyanuric Acid-Related Recall}

The most recent pet food recall due to a chemical contaminant/adulterant also turned out to be the largest in
US history [12]. It was triggered by adulteration of dog and cat food with industrial chemicals melamine and cyanuric acid. On March 16, 2007, a recall was initiated which involved 60 brands of dog and cat foods manufactured by Menu Foods, Inc. due to presence of melamine and cyanuric acid which had been intentionally and fraudulently added to wheat gluten by Chinese suppliers. Wheat gluten is an ingredient used in the manufacture of wet foods. About 53,373,600 cans or pouches of dog and cat food across the USA and Canada were involved in this recall. Menu Foods subsequently expanded the recall to other food brands. At the end of the crisis over 100 brands of dog and cat foods were involved in the recall. Consumer complaints to the firm indicated that cats and dogs were getting sick or dying after eating wet "cuts and gravy" food products. The cardinal effect of this toxicosis was acute renal failure in both cats and dogs [12]. Following an extensive investigation by the FDA, it was discovered that adulteration by melamine and cyanuric acid were the cause of the problem. In this case, the FDA provided evidence of analytical results confirming presence of melamine and cyanuric acid in adulterated pet food. The total estimated number of dogs that became ill, died or were euthanized due to this toxicosis is hard to estimate, but hundreds have been identified [4].

Melamine is an industrial chemical used for a variety of purpose including fertilizer, flame retardant and in the manufacturing of resins and adhesives. Adding melamine to foods falsely increases protein content. In the case of this pet food recall, melamine was intentionally and illegally added to wheat gluten in China. The adulterated wheat gluten was exported to North America and used in manufacture of pet food. The melamine was contaminated with cyanuric acid. In pets, melamine or cyanuric acid alone are largely nontoxic but when present in combination there is a synergistic effect on renal toxicity [37,38]. There are several publications on the melamine pet food contamination of 2007 [4, 37-40]. Melamine is also toxic in humans as demonstrated by illness in about 60,000 children and infants and at least six deaths following consumption of melamine-contaminated baby food products in China [41].

The difference between the melamine adulteration and other pet food recalls is that melamine was intentionally added to foods to defraud consumers. This kind of adulteration is challenging for pet food firms and may be impossible to avoid $100 \%$ of the time given there are tens of thousands of potentially toxic chemicals in the world. The fact that this kind food contamination occurred is a warning to the industry and food industry regulators that this kind of adulteration is likely to occur and can involve any chemical or toxicant being added to pet food or pet food ingredients for different reasons. As such, the industry needs to put in place measures to prevent this kind of 
adulteration in pet foods. One positive outcome from the melamine pet food recall is that the FDA has set a safety guideline of $2.5 \mathrm{ppm}$ for melamine and melamine-related compounds in food [42]. Any food item containing more than $2.5 \mathrm{ppm}$ of melamine and melamine-related compounds is considered adulterated.

\section{Discussion}

The objective of this review was to describe the pet food recall procedure from start to finish, and to review class I and II pet food recalls from 1996 to 2008, with a specific focus on those due to chemical contaminants/adulterants. Pet food in the US is generally safe and as results of this study have shown, only six recalls were due to chemical adulteration over the entire review period. Five of the six recalls were one-firm recalls only and did not cause as many challenges as the 2007 recall involving melamine and cyanuric acid which involved multiple firms in USA and Canada. The 2007 recall was a challenge because it took at least 3 weeks before the causal adulterants were identified, and even longer for federal regulators and scientific community to prove that indeed it was the interaction between melamine and cyanuric acid that was responsible for the acute renal failure in cats and dogs. That recall also exposed significant deficiencies in the recall process which have been addressed by the enactment of the FDAAA of 2007. The responsibility of producing safe food rests with the manufacturer (firm). In all recalls discussed in this review the firms involved voluntarily initiated the recalls. In all cases, adulteration was proven by chemical analysis either by the firms and/or by the FDA. In addition, supportive clinical signs of intoxication consistent with adulterant were confirmed by FDA experts and documented in the FDA health hazard evaluation reports. The new FDAAA of 2007 when fully implemented will make it easier and clearer for consumers to report adverse food events, enhance communication between the FDA and states, and critically reduce the time firms take to report reportable foods to the FDA. This will enhance protection of consumers and their pets. However, because firms have to report adverse food events to the FDA within $24 \mathrm{~h}$ of knowing about then, there is a possibility that firms will be on the edge and likely report "adverse food events" even when consumer complaints may be baseless or not fully confirmed. Whereas this new approach is likely to save guard pet and public safety better in case of true adulterations, caution should be exercised by all those concerned in using client's complaints data and/or incomplete clinical data to run risk assessments and HHE. Hasty recalls based on incomplete data that may later turn out to be faulty may be very costly to firms both financially and by negatively impacting the credibility of the firm and regulatory authorities. Thus a healthy balance needs to be reached to protect both pet and public safety and interests of the firm.

There is no system which can be considered perfect, and certainly the recall procedure will likely continue to have some shortcomings. For example, many states in the union do not have laws that treat food adulterants or chemicalinduced food-borne diseases as reportable diseases. Besides, some of the states which have such laws, the laws are old and may not meet modern day challenges. As such, a chemical-induced food-borne disease may go unreported in some states. Such gaps need to be addressed. Secondly, some veterinary practitioners may not be familiar with both federal and state reporting requirements and procedures. These issues need to be addressed to continue to improve the food recall process.

\section{Conclusion}

Overall, pet food manufactured in the USA is safe for pets. This review of pet food chemical contaminants indicates that pet food adulteration does occur. For the period covered in this review, pet food recalls involved aflatoxin, a mycotoxin; vitamin $\mathrm{D}_{3}$, an essential dietary vitamin; methionine, an amino acid; and melamine/cyanuric acid, both industrial chemicals. The causes of adulteration included inadequate testing of raw materials for toxins, use of wrong or faulty mixing equipment, and misformulation of raw materials. Effort needs to be made by firms to limit these and other potential sources of pet food contamination to safeguard pet and public safety. The true extent of pet diseases caused by consumption of contaminated pet food is not clear, as there was no agency tracking adverse events in pets caused by food adulteration. However, when the new FDAA Act of 2007 is fully implemented this will likely change as the new law calls for tracking such cases. More work needs to be done at state level, for example, to include chemical-induced food-borne diseases as reportable diseases. This will prevent some cases from going unreported.

\section{References}

1. Humane Society of the United States (2009). U.S. pet ownership statistics: American Pet Products Manufacturers Association 20092010 National Pet Owners Survey. http://www.humanesociety.org/ issues/pet_overpopulation/facts/pet_ownership_statistics.html

2. Pet Food Institute (2010). U.S. Pet Food Tonnage Produced. http:// www.petfoodinstitute.org/Index.cfm?Page=USPetFoodTonnageData

3. Dzanis DA (2008) Anatomy of a recall. Top Companion Anim Med 23(3):133-136 
4. Rumbeiha WK, Agnew D, Maxie G, Hoff B, Page C, Curran P, Powers B (2010) Analysis of a Survey Database of Pet FoodInduced Poisoning in North America. J Med Toxicol 6:172-184

5. Kempson KM, Keenan DP, Sadani PS et al (2002) Food management practices used by people with limited resources to maintain food sufficiency as reported by nutrition educators. J Am Diet Assoc 102(12):1795-1799

6. Title 21 Code of Federal regulations Chapter 1 Part 7. Washington DC, Government Printing Office, 2007

7. Food and Drug Administration Amendment Act, Title X, Food Safety 2007.

8. Health Hazard Evaluation November 27, 1998: recall No. 99003R. Food and Drug Administration, Department of Health and Human Services. Obtained through the Freedom of Information Act.

9. Health Hazard Evaluation January 13, 2000: recall no. 0019R. Food and Drug Administration, Department of Health and Human Services. Obtained through the Freedom of Information Act.

10. Health Hazard Evaluation April 10, 2006: firm-initiated recall of Royal Canin Veterinary Diet ${ }^{\mathrm{TM}}$ products with excessive amounts of vitamin $\mathrm{D}_{3}$. Food and Drug Administration, Department of Health and Human Services. Obtained through the Freedom of Information Act.

11. Health Hazard Evaluation February 28, 2001: firm initiated recall (The IAMS Company). Food and Drug Administration, Department of Health and Human Services. Obtained through the Freedom of Information Act.

12. Health Hazard Evaluation November 14, 2007: dog food contaminated with melamine and cyanuric acid. Food and Drug Administration, Department of Health and Human Services. Obtained through the Freedom of Information Act.

13. Stenske KA, Smith JR, Newman SJ et al (2006) Aflatoxicosis in dogs and dealing with suspected contaminated commercial foods. J Am Vet Med Assoc 228(11):1686-1691

14. Newman SJ, Smith JR, Stenske KA et al (2007) Aflatoxicosis in nine dogs after exposure to contaminated commercial dog food. J Vet Diagn Invest 19:168-175

15. Dereszynski DM, Center SA, Randolph JF et al (2008) Clinical and clinicopathologic features of dogs that consumed food borne hepatotoxic aflatoxins: 72 cases (2005-2006). J Am Vet Med Assoc 232(9):1329-1337

16. Boermans HJ, Leung MCK (2007) Mycotoxins and the pet food industry: toxicological evidence and risk assessment. Int J Food Microbiol 119:95-102

17. Liggett AD, Colvin BM, Beaver RW et al (1986) Canine aflatoxicosis: a continuing problem. Vet Hum Toxicol 28(5):428-430

18. Food and Drug Administration (2009). Compliance Policy Guides section 683.100 action levels for aflatoxins in animal feeds. http:// www.fda.gov/ICECI/ComplianceManuals/CompliancePolicyGui danceManual/ucm074703.htm

19. Rumbeiha WK (2001) Clinical implications of mycotoxicosis in companion animals. In: Lyons TP, Jacques KA (eds) Science and Technology in the Feed Industry: proceedings of Alltech's 17th annual symposium. Nottingham University Press, United Kingdom, pp 225-233

20. International Agency for Research on Cancer. Monographs on the evaluation of the carcinogenic risk of chemicals to man. Geneva: World Health Organization, International Agency for Research on Cancer, 1972-Present 1993;56:362.

21. Krishnamachari KA, Nagaarajan V, Bhat RV et al (1975) Hepatitis due to aflatoxicosis: an outbreak in Western India. Lancet 305:1061-1063

22. Ngindu A, Kenya PR, Ocheng DM et al (1982) Outbreak of acute hepatitis by aflatoxin poisoning in Kenya. Lancet 319:1346-1348
23. Centers for Disease Control and Prevention. Outbreak of aflatoxin poisoning- Eastern and Central Provinces, Kenya, January-July 2004. Morbidity and Mortality Weekly Report September 3, 2004 53(34);790-793.

24. Willis RM, Mulvihill JJ, Hoofnagle JH (1980) Attempted suicide with purified aflatoxin. Lancet 315(8179):1198-1199

25. Williams JH, Phillips TD, Jolly PE et al (2004) Human aflatoxicosis in developing countries: a review of toxicology, exposure, potential health consequences, and interventions. Am J Clin Nutr 80:1106-1122

26. Rumbeiha WK, Fitzgerald SD, Kruger JM et al (2000) Use of pamidronate disodium to reduce cholecalciferol-induced toxicosis in dogs. Am J Vet Res 61(1):9-13

27. National Research Council (2006) Nutrient requirements of dogs and cats. National Academies Press, Washington, D.C, pp 203 204

28. Anonymous, Institute of Medicine, Food and Nutrition Board. Dietary reference intakes: calcium, phosphorus, magnesium, vitamin D, and fluoride. Washington, DC: National Academy Press, 1997.

29. Ekperigin HE, Vora P (1980) Histological and biochemical effects of feeding excess dietary methionine to broiler chicks. Avian Dis 24(1):82-95

30. Association of American Feed Control Officials. 2008 Official Publication.

31. Fau D, Peret J, Hadjiisky P (1988) Effects of ingestion of high protein or excess methionine diets by rats for two years. J Nutr 118(1):128-133

32. Biourge VC, Pierson P, Metz F. 2002. Methionine toxicosis in a group of 7 hunting dogs. Proc Am Acad Vet Nutr Res Symp Dallas, TX. May 29. pp. 9-10.

33. Fau D, Smalley KA, Rogers QR et al (1987) Effect of excess dietary methionine on weight gain and plasma amino acids in kittens. J Nutr 117:1838-1843

34. Troen AM, Lutgens E, Smith DE et al (2003) The atherogenic effect of excess methionine intake. P Natl Acad Sci USA 100 (25):15089-15094

35. McCully KS (1969) Vascular pathology of homocysteinemia: implications for the pathogenesis of arteriosclerosis. Am J Pathol 56(1):111-128

36. Garlick PJ (2006) Toxicity of methionine in humans. J Nutr 136:1722s-1725s

37. Puschner B, Poppenga RH, Lowenstine LJ et al (2007) Assessment of Melamine and Cyanuric Acid Toxicity in Cats. J Vet Diagn Invest 19(6):616-624

38. Brown CA, Jeong KS, Poppenga RH, Puschner B, Miller DM, Ellis AE et al (2007) Outbreaks of renal failure associated with melamine and cyanuric acid in dogs and cats in 2004 and 2007. J Vet Diagn Invest 19(5):525-531

39. Burns K (2007) Events leading to the major recall of pet foods. J Am Vet Med Assoc 230(11):1600-1602

40. Cianciolo RE, Bischoff K, Ebel JG et al (2008) Clinicopathologic, histologic, and toxicologic findings in 70 cats inadvertently exposed to pet food contaminated with melamine and cyanuric acid. J Am Vet Med Assoc 233(5):729-737

41. Anonymous, World Health Organization. Toxicological and health aspects of melamine and cyanuric acid: report of a WHO expert meeting in collaboration with FAO, supported by Health Canada, Ottawa, Canada, 1-4 December 2008. Geneva: WHO Press; 2009.

42. Food and Drug Administration (2008). FDA issues interim safety assessment of melamine and melamine-related compounds in food. http://www.fda.gov/NewsEvents/Newsroom/PressAnnouncements/ 2008/ucm116960.htm 